\title{
The Evolution of Passive Galaxies since $z=1$ : Major Mergers vs Secular Processes
}

\author{
Carlos López-Sanjuan ${ }^{1,2,3}$, Marc Balcells ${ }^{1,2,4}$, Pablo G. \\ Pérez-González $^{5}$, Guillermo Barro ${ }^{5}$, César Enrique García-Dabó ${ }^{1,6}$, \\ Jesús Gallego ${ }^{5}$ \& Jaime Zamorano ${ }^{5}$
}

\author{
${ }^{1}$ Instituto de Astrofísica de Canarias, \\ Calle Vía Láctea s/n, E-38205 La Laguna, Tenerife, Spain \\ email: carlos.lopez@oamp.fr
}

${ }^{2}$ Departamento de Astrofísica, Universidad de La Laguna, E-38200 La Laguna, Tenerife, Spain

${ }^{3}$ Laboratoire d'Astrophysique de Marseille,

38, rue Frédéric Joliot-Curie, F-13388 Marseille cedex 13, France

${ }^{4}$ Isaac Newton Group of Telescopes, Aptdo. Correos 321, E-38700 Santa Cruz de La Palma, Tenerife, Spain

${ }^{5}$ Departamento de Astrofísica y Ciencias de la Atmósfera, Facultad de C.C. Físicas, Universidad Complutense de Madrid, E-28040 Madrid, Spain

${ }^{6}$ FRACTAL SLNE, C/ Tulipán 2, portal 13, 1 A, E-28231 Las Rozas, Madrid, Spain

\begin{abstract}
We study the evolution of the red sequence and the blue cloud since $z \sim 1$ to present in GOODS-S field for $M_{B} \leqslant-20$ and $M_{\star} \geqslant 10^{10} M_{\odot}$ selected galaxies. We segregate the galaxies in early-type galaxies (ET, E/S0/Sa), and late-type galaxies (LT, Sb-Irr) by their position in the concentration-asymmetry plane, while in passive and star-forming by their rest-frame $N U V-R$ color and their $24 \mu \mathrm{m}$ properties.

We find that red sequence (passive early types) comoving number density rise with cosmic time in both samples, while blue cloud (star-forming late types) number density descent in luminosity-selected samples and increase in mass-selected sample. We also find a third population that comprises star-forming early-type galaxies.

Comparing the observed evolution with the disk-disk (wet) major merger rate in GOODS-S, we infer that only $\sim 20 \%$ of $M_{\star} \geqslant 10^{10} M_{\odot}$ red sequence galaxies appeared since $z=1$ can be explained by this kind of mergers, suggesting that minor mergers and secular evolution are the main processes in the red sequence evolution of $M_{\star} \geqslant 10^{10} M_{\odot}$ galaxies since $z \sim 1$.
\end{abstract}

Keywords. galaxies: evolution, galaxies: formation, galaxies: interactions

\section{Introduction}

It is known that more massive galaxies are the first to form their stars and populate the mass function, being in place at $z \sim 1$. This is the called "downsizing" scenario (Cowie et al. 1996): several massive galaxies experienced most of their star formation at early times and were passive by $z \sim 1$, and many among the less massive galaxies experienced extended star-formation histories (Bundy et al. 2006; Pérez-González et al. 2008). This fact is a challenge to the popular hierarchical $\Lambda$-CDM models, in which more massive dark matter halos are the final stage of successive mergers of smaller halos. However, the treatment of the barionic component is still unclear and only accessible via models (e.g. Bower et al. 2006). In this framework, the role of galaxy mergers in the buildup of the red sequence and their relative importance in the evolution of the galaxy properties, e.g. morphology, mass, and color, is an important open question. 

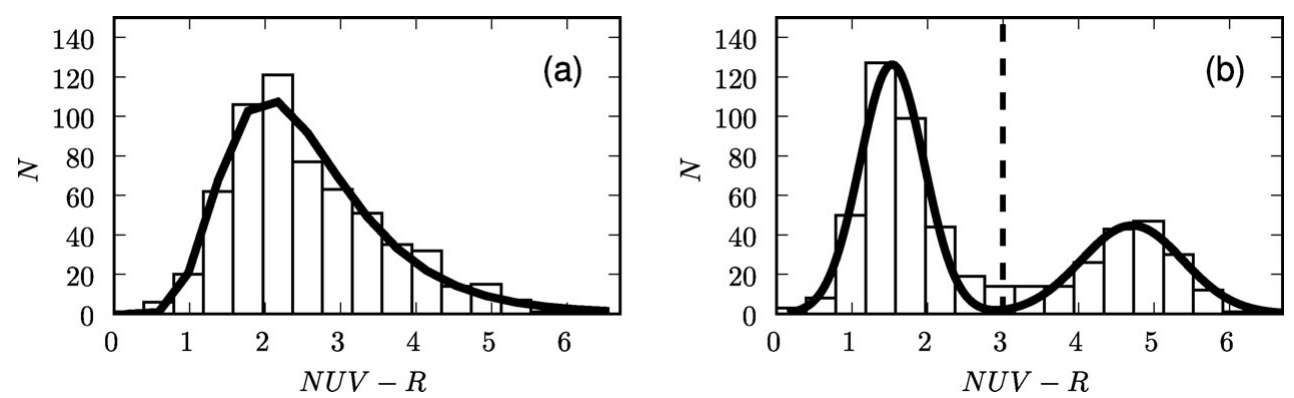

Figure 1. Rest-frame $N U V-R$ histograms of galaxies with $0.2<z<1.1$ and $M_{B} \leqslant-19.5$. Those galaxies with detection in $24 \mu \mathrm{m}$ (dusty galaxies) are in panel (a), while those without detection in $24 \mu \mathrm{m}$ are in panel (b). Black solid lines are the best fit to the data with (a) a lognormal distribution, (b) two gaussians. The black dashed line marks the separation between blue and red galaxies, $N U V-R=3$.

In this work we summarize preliminary results about the redshift evolution of the red sequence and the blue cloud in $B$-band luminosity- and mass-selected samples up to $z \sim 1$ in GOODS-S area, and explore the role of disk-disk (wet) major mergers in this evolution. We use $H_{0}=70 \mathrm{Km} \mathrm{s}^{-1} \mathrm{Mpc}^{-1}, \Omega_{M}=0.3$, and $\Omega_{\Lambda}=0.7$ throughout. All magnitudes are Vega unless noted otherwise.

\section{Data}

Galaxies were selected from the merged photometric catalog of Spitzer/IRAC selected sources in GOODS-S published by Pérez-González et al. (2008). This catalog has 9676 IRAC sources detected in $225 \operatorname{arcmin}^{2}$. The catalog is $75 \%$ complete down to [3.6] $\sim 23.5$ $(\mathrm{AB})$, and includes spectroscopic redshifts and photometric data in a variety of bands from the UV to the MIR, including GALEX(UV), HST/ACS $(F 435 W, F 606 W, F 775 W$, $F 850 L P), V L T /$ ISAAC $(J, H, K)$, Spitzer/IRAC $(3.6,4.5,5.4,8.5 \mu \mathrm{m})$, Spitzer/MIPS $(24 \mu \mathrm{m}), C H A N D R A$ (X-Ray) and COMBO-17 filters. We have used the photometric redshifts, absolute magnitudes, stellar masses, and star-formation rates presented in Pérez-González et al. (2008)†. We took two different samples: one comprises 1122 sources with $M_{B} \leqslant-20$, while another comprises 982 with $M_{\star} \geqslant 10^{10} M_{\odot}$.

\section{Defining the red sequence and the blue cloud}

To define the different populations in our samples we study two main properties of galaxies: their strucute and their star formation. In the former, we segregate galaxies by their position in the concentration - asymmetry plane $(C-A$ plane $)$, in which galaxies present a clear bimodality, into early-type galaxies (E/S0/Sa, ET hereafter), late-type galaxies (Sb-Irr, LT hereafter), and disk-disk major merger remnants, which have high asymmetry values. All the details about the structural evolution of GOODS-S galaxies are in López-Sanjuan et al. (2009c), while the disk-disk major mergar rate is extensively studied in López-Sanjuan et al. (2009b). For a complete description of the major merger fraction determination, see also López-Sanjuan et al. (2008) and López-Sanjuan et al. (2009a).

To classify our galaxies as passive (i.e., without or with residual star formation) or starforming (SF), we split our catalog in two subsamples: the first comprises the catalog's

$\dagger$ See http://t-rex.fis.ucm.es/ pgperez/Proyectos/databaseuse.en.html, for details. 

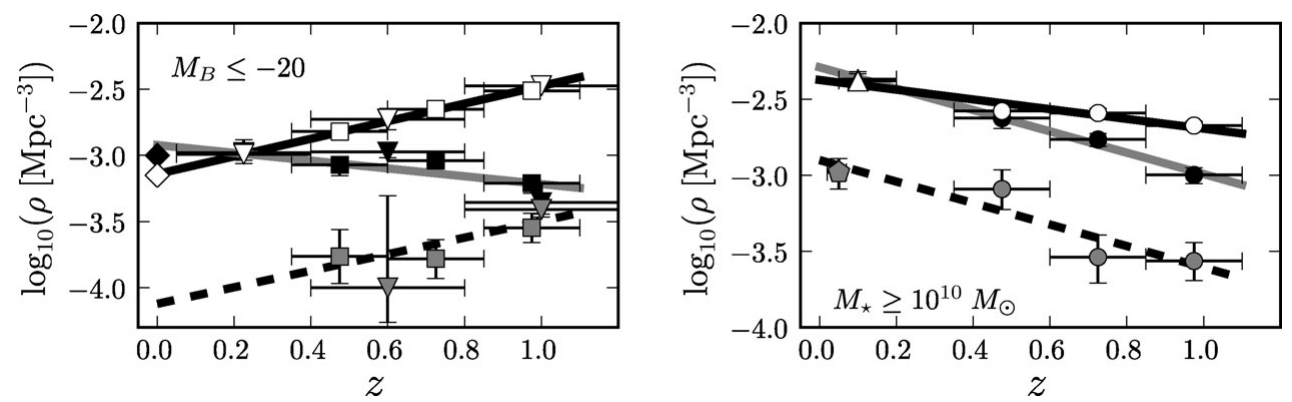

Figure 2. Comoving number density of red sequence (black symbols), blue cloud (white symbols), and SFET galaxies (gray symbols) vs redshift for $M_{B} \leqslant-20$ (left) and $M_{\star}>10^{10} M_{\odot}$ galaxies (right). Squares and circles are from present work, inverted triangles from Ilbert et al. (2006), diamonds from López-Sanjuan et al. (2009c), triangles from Mandelbaum et al. (2006), and the pentagon from Gadotti (2009). Lines are the linear fits to the data.

sources detected in $24 \mu \mathrm{m}$ by Spitzer/MIPS, white the second comprises those galaxies without $24 \mu \mathrm{m}$ detection. The former are dusty SF galaxies (panel [a] in Fig. 1), while the latter are either galaxies without star formation or SF without enought dust to be detected in $24 \mu \mathrm{m}$. To break the last degeneracy, we use the rest-frame $N U V-R$ color, in which galaxies without $24 \mu \mathrm{m}$ detection define two populations: the blue galaxies $(N U V-R \leqslant 3, \mathrm{SF}$ without enought dust) and the red galaxies $(N U V-R>3$, without star formation, panel [b] in Fig. 1). Finally, we take red galaxies as passive, while dusty and blue as SF.

Now we can deffine four galaxy populations: passive early-type galaxies (red sequence hereafter), star-forming late-type galaxies (blue cloud hereafter), star-forming early-type galaxies (SFET hereafter), and passive late-type galaxies.

\section{The evolution of the red sequence and the blue cloud}

We show in Fig. 2 the evolution with redshift of the comoving number density of the red sequence, the blue cloud, and the SFET galaxies, both for $M_{B} \leqslant-20$ galaxies (left panel), and for $M_{\star} \geqslant 10^{10} M_{\odot}$ galaxies (right panel). The number density of passive late types is not significant, so we do not show for clarity.

We find that, in $B$-band luminosity-selected sample, the comoving number density of star-forming galaxies (i.e. blue cloud and SFET) descent with cosmic time. In addition, SFET galaxies are an order of magnitude less abundant that blue cloud systems: since interacting systems only accounts for $20-30 \%$ of star formation density at these redshifts (e.g. Jogee et al. 2009), the bulk of star formation is located in the blue cloud. Because $B$-band luminosity is dominated by the young stellar component in these galaxies, the number density descent refects the fading of the star formation since $z \sim 1$ (e.g. Villar et al. 2008). On the other hand, red sequence increase its number density a factor of two.

The behaviour is different in the mass-selected sample: the comoving number density of the three populations grows with cosmic time; the blue cloud a factor of two, while early types (i.e. red sequence and SFET) a factor of five. Combinig luminosity and mass trends, we infer that we need a transformation form the blue cloud, in which stars are forming, and the red sequence, in which stellar mass reside, in agreement with Bell et al. (2007). The question is, can disk-disk major mergers account for the observed evolution in red sequence? 


\section{The role of major mergers in red sequence evolution since $z=1$}

We compare the evolution in red sequence at $0<z \leqslant 1$ from the previous section with the number density of disk-disk (wet) major merger remnants in the same redshift range. We study this kind of mergers because are able to transform blue clod galaxies in red sequence ones (e.g Naab et al. 2006). We use López-Sanjuan et al. (2009b) wet major merger rates in the comparison, obtaining tha only $\sim 20 \%$ of the red sequence evolution since $z \sim 1$ can be explained by wet major mergers, in agreement with the results from Bundy et al. (2009), de Ravel et al. (2009), or Wild et al. (2009).

We suggest minor mergers and secular processes to explain the other $\sim 80 \%$ in red sequence evolution. We called this the "slow path": blue cloud galaxy (e.g., Sc) $\rightarrow$ SFET $(\mathrm{Sa}) \rightarrow$ red sequence galaxy (Sa/S0); in opposition to the "fast path" driven by wet major mergers: two blue cloud galaxies $\rightarrow$ one red sequence galaxy (S0/E). This picture is consistent with the evolution of SFET in previous section: we propose that these systems are the Sa transition between the blue cloud and the red sequence in "slow path", a role also suggested by other authors (e.g. Cameron et al. 2009).

Studies in higher samples and the determination of structure in redder bands, more relates with the stellar mass of the galaxy than $B$-band, are needed to understand better the transition from the blue cloud to the red sequence in the last 8 Gyr of the Universe.

\section{Acknowledgements}

We dedicate this work to the memory of our six IAC colleagues and friends who met with a fatal accident in Piedra de los Cochinos, Tenerife, in February 2007, with a special thanks to Maurizio Panniello, whose teachings of python were so important for this work. We thank G. Bruzual for a stimulating and challenging symposium. This work was supported by the Spanish Programa Nacional de Astronomía y Astrofísica through project number AYA2006-12955.

\section{References}

Bell, E. F., Zheng, X. Z., Papovich, C. et al. 2007, ApJ, 663, 834

Bower, R. G., Benson, A. J., Malbon, R. et al. 2006, MNRAS, 370, 64

Bundy, K., Ellis, R. S., Conselice, C. J. et al. 2006, ApJ, 651, 120

Bundy, K., Fukugita, M., Ellis, R. S. et al. 2009, ApJ, 697, 1369

Cameron, E., Driver, S. P., Graham, A. W. et al. 2009, ApJ, 699, 105

Cowie, L. L., Songaila, A., Hu, E. M., \& Cohen, J. G. 1996, AJ, 112, 839

de Ravel, L., Le Fèvre, O., Tresse, L. et al. 2009, A\&A, 498, 379

Gadotti, D. A. 2009, MNRAS, 393, 1531

Ilbert, O., Lauger, S., Tresse, L. et al. 2006, A\&A, 453, 809

Jogee, S., Miller, S. H., Penner, K. et al. 2009, ApJ, 697, 1971

López-Sanjuan, C., García-Dabó, C. E., \& Balcells, M. 2008, PASP, 120, 571

López-Sanjuan, C., Balcells, M., García-Dabó, C. E. et al. (2009a), ApJ, 694, 643

López-Sanjuan, C., Balcells, M., Pérez-González, P. G. et al. (2009b), A\&A, 501, 505

López-Sanjuan, C., Balcells, M., \& Pérez-González, P. G. et al. (2009c), ApJ, submitted

Mandelbaum, R., Seljak, U., Kauffmann, G. et al. 2006, MNRAS, 368, 715

Pérez-González, P. G., Rieke, G. H., Villar, V. et al. 2008, ApJ, 675, 234

Naab, T., Jesseit, R., \& Burkert, A. 2006, MNRAS, 372, 839

Villar, V., Gallego, J., Pérez-González, P. G. et al. 2008, Apj, 677, 169

Wild, V., Walcher, C. J., Johansson, P. H. et al. 2009, MNRAS, 395, 144 